\title{
Susceptibility of European pear cultivars to Venturia pirina Aderh. infection at a variety collection in Hungary
}

\author{
Benedek, P. ${ }^{1}$, Szabó, T. ${ }^{2}$, Szabó, Z. ${ }^{3}$ \& Nyéki, J. ${ }^{3}$ \\ ${ }^{1}$ University of West Hungary, Faculty of Agricultural ad food Sciences, \\ H-9200 Mosonmagyaróvár, Vár 4. (e-mail: benedek@mtk.nyme.hu) \\ ${ }^{2}$ Research and Extension Centre for Fruit Growig, H-4244 Úfehértó, Vadastag 2. \\ ${ }^{3}$ Institute of Extension and Development, University of Debrecen, H-4032 Debrecen, Böszörményi str. 138.
}

\begin{abstract}
Summary: Field sensitivity of 271 European pear genotypes were evaluated in a large variety collection of pear in Hungary during a nine years survey. Substantial differences were detected in the rate of pear scab infection of leaves and fruits. Some 18 per cent of the inspected genotypes remained completely free of infection even in the years with weather favouring to the disease. On the other hand, $78 \%$ of the inspected genotypes were infected in some or more extent. The rate of infection was generally higher on leaves than on fruits. $18.6 \%$ of the genotypes inspected were tolerant to leaf infection and their majority $(81.1 \%)$ were tolerant to fruit infection. Some one fifth of the genotypes (19.7\%) were highly susceptible to leaf infection but only minor rate of them $(2.2 \%)$ were highly susceptible to fruit infection. There were 44 genotypes that could be regarded as tolerant to pear scab leaf and fruit infection under field conditions. Some of these genotypes may be suitable to commercial pear growing with highly reduced chemical control against pear but their yield capacity and fruit quality should be evaluated.
\end{abstract}

Key words: pear genotypes, pear scab, susceptibility, resistance

\section{Introduction}

Some European pear (Pyrus communis L.) cultivars are known to be less sensitive to pear scab (Venturia pirina Aderh.) infestation than others for many decades. For example Husz (1941) in Hungary stated more than 70 yeas ago that the few cultivars (Bosc kobak, Clairgeau vajkörte, Papkörte, Serres Olivér) were scarcely susceptible to this disease and a number of other cvs at the same time were greatly sensitive to pear cab (Nyári arabitka, Hardenpont vajkörte, Napóleon vajkörte, Diel vajkörte, Avranchesi jó Lujza, Liegel vajkörte, Téli esperes, Nyári Kálmán, Erdei vajkörte, Vilmos, Kis muskotály). This problem has been very important in commercial pear growing and for this reason several field studies have been made later in many countries to explore further differences between the sensitivity of pear cultivars against Venturia pirina. The most extensive field studies were made by Postman et al. (2004) who evaluated the susceptibility against pear scab of as much as some 200 European pear cultivars for as long time as 10 years in the pear gene bank of the USDA-ARS at Corvallis, Oregon. They also studied the sensitivity of artificially infected seedlings in glasshouse tests. They stated that 38 per cent of the inspected European pear cultivars were regularly infected in a great extent while some 8 per cent of them remained almost free of infection or were negligibly infected only. They discovered some European pear cultivars that can be grown profitably in the US with no fungicide treatment against pear scab (Arganche, Batjarka, Brandy, Erabasma, Muscat, Passe Crassane).
It is also known for a long time that Asian pear (Pyrus pyrifolia Burm. f. Nak.) is definitely resistant against pear scab (see for example Postman et al., 2004; Villata et al., 2005). Also the interspecific hybrids of European X Asian pears are generally immune to pear scab infections (Villata et al., 2005; Brewer et al., 2009). The degree of resistance of intesrpecific hybrids seems to be dependent on the ratio of Asian pear genome in the hybrids. The resistance is usually almost complete when the ratio of Asian pear genome is at least 50 per cent but the hybrids are almost completely susceptible when the ratio of Asian pear is not more than 1225 per cent in their genomes (Brewer et al., 2009).

Comparing the reactions to pear scab infections of one highly susceptible (Angelys) and one definitely resistant pear cultivar (Navara) Chevalier et al. (2011) have shown that the leaves of the resistant cultivar produce hypersensitive reactions to the pear scab infections because local necroses appear around the place of fungal attacks while the fungus can successfully penetrate into the leave tissues and finally sporulation is taking place in the leaves of the susceptible cultivar. Bouvier et al. (1912) have discovered one resistance gene in the genome of the resistant cultivar 'Navara' that is responsible for the hypersensitive necroses and they noted this as Rvp1 gene. This gene is located at the same genomic region of the pear where the scab resistant gene can be found in the genome of scab resistant apples. The Rvp1 resistance gene can be mapped close to the $\mathrm{CH} 02 \mathrm{~b} 10$ microsellite marker in the second linkage group of pear genome so developing a genetic marker based method seems to be promising to detect 
resistant pear genotypes in breeding resistant pear cultivars (Chevalier et al., 2011).

So it can bee seen, that very promising research is going on in many countries to find out pear genotypes being resistant to pear scab infections. In Hungary related research was made by Szabó and Soltész (1998) who carried out field observations in the largest Hungarian pear gene bank at the Research and Extension Centre for Fruit Growing at Újfehértó. They made systematic observations on field infection of more than 200 pear genotypes for 10 years between 1986 and 1996 and. Finally the susceptibility of 126 pear genotypes to pear scab infections were evaluated, and the majority of these were regarded to be highly sensitive but some 13.6 per cent of the inspected genotypes were less susceptible. However, all genotypes that received less than 10 per cent leaf infection during the ten years' observation period were regarded as 'less susceptible' in their study. Unfortunately this category can consist of both 'resistant' or 'tolerant' and 'less sensitive' (or 'slightly susceptible') cultivars, too. For this reason we decided to carry out long term field investigations at the same place to establish if there are any genotypes in this gene bank that can be supposed to be 'tolerant' under field conditions with no scab infections in long term studies.

\section{Material and method}

Our long term field investigations were made in the largest gene bank of pear in Hungary that situated in the experimental area of the Research and Extension Centre for Fruit Growing at Újfehértó (North-Eastern Hungary). The gene bank was planted at a sandy area being typical to the region. There are 486 pear genotypes in the collection. We made systematic field observations on pear scab incidence at 271 genotypes the trees of that were at least 10 years old in 1996 when our work started. Younger trees were not included in the research. Trees were medium sized; most of them were grafted to quince as rootstock. The investigated genotypes consisted of commercial cultivars, conventional land varieties, registered local clones, seedlings as well as wild seedlings.

Investigations were made during two periods of time, the first period was from 1996 to 1999 (four years) and the second period from 2004 to 2008 (five years). This is nine years altogether. Most genotypes were observed all along the nine years, but a part of them was neglected for one year and some of them for two years for the lack of necessary manpower. So, most genotypes were observed during 9 years, some ones for 8 and a portion of them for 7 years.

The gene bank received a mild, integrated plant protection program all along the experimental period; it received greatly reduced pesticide applications with pesticides being relatively safe to the environment. The pesticides applied changed slightly during the long period of experimentation because pesticide regulations changed meanwhile for EU decisions.
The trees were carefully evaluated for pear scab (Venturia pirina) infection in mid-August each year because this is the top period of pear scab infection in this region. We counted the rate of infection on leaves and fruits separately at the four points of the compass in the crowns of each tree using a five grade 'infection scale' as follows:

\section{a: no infection}

b: ratio of infected leaves of fruits is $1-10 \%$

c: ratio of infected leaves of fruits is $10-20 \%$

$\mathrm{d}$ : ratio of infected leaves of fruits is $20-50 \%$

e: ratio of infected leaves of fruits is $50-70 \%$

f: ratio of infected leaves of fruits is $70-100 \%$

Evaluating the results we calculated the mean infection levels of leaves and fruits separately of each inspected genotype for the two periods of investigations (1996-1999, 2004-2008, resp.) and for the total time of observations. However, pear scab infection was very low is some years because summer weather was unfavourable (much less rain than average) to pear scab incidence and so majority of the observed pear genotypes remained free of infection (1996, 1997, 1999, 2005, 2005). There were four other years when the weather favoured to pear scab incidence and so substantial differences appeared in the scab infection of the observed pears $(1998,2004,2006,2008)$. In these years a number of inspected genotypes were infected by pear scab more or less but some of then remained free of infection even in these periods. So the scab incidence in these years was taken into account to characterise the susceptibility of inspected genotypes to pear scab infection. Taking the scab infection levels into account the inspected genotypes were classified. These categories were established for the purpose to characterise the susceptibility of inspected pear genotypes to pear scab incidence. So, the susceptibility (typical rate of infection) of leaves and fruits were evaluated separately according to the following categories:

0 . Tolerant (free of infection): No infection on leaves and fruits during the 9 years of observations including the fours years with weather favouring pear cab incidence (scale of infection was always 'a' during 9 years).

1. Slightly susceptible (slight infection is typical): Leaves or fruits were infected only in years with weather favouring pear scab incidence but the infection was lower than 10 per cent in all years including the fours years with weather favouring the disease (scale of infection was maximum 'b' during 9 years).

2. Susceptible (slight or medium infection is typical): Leaves or fruits were infected at least in the years with weather favouring pear scab incidence and the infection was more than 10 per cent but it was less than 50 per cent including the fours years with weather favouring the disease (scale of infection was maximum 'c-d' during 9 years).

3. Highly susceptible (strong infection is typical): Leaves or fruits were infected at least in the years with weather favouring pear scab incidence and the infection was 
higher than 50 (sometimes close to 100) per cent in the years with weather favouring the disease incidence (scale of infection was always 'e-f' in the years with weather favouring the disease).

\section{Results}

Tale 1 demonstrates the rate of relative pear scab infection of the 271 investigated pear genotypes. In can be seen that in spite of the mild, integrated plant protection program applied at the inspected pear gene bank lower or higher infection has developed at the majority of the evaluated genotypes in those years when favourable weather has encouraged the infection. Simultaneously, a smaller number of genotypes have remained completely free of pear scab infection even in the years with weather favourable to pear scab (Table 1). For this reason our field observations have made it possible to compare the sensitivity pear genotypes to pear scab infections.

Table 1. Average Venturia pirina field infection of European pear genotypes at the 'Újfehértó' pear gene bank (Hungary), in the four years with favourable weather to disease incidence (1998, 2004, 2006, 2008), during the nine years period between 1996-1999 and 2004-2008.

\begin{tabular}{|c|c|c|}
\hline \multirow[b]{2}{*}{ Cultivar } & \multicolumn{2}{|c|}{ Rate of infection* } \\
\hline & $\begin{array}{l}\text { on the } \\
\text { leaves }\end{array}$ & $\begin{array}{l}\text { on the } \\
\text { fruits }\end{array}$ \\
\hline Augusztusban érő piros lapított (18/5) & 2 & $\mathbf{0}$ \\
\hline Augusztusi piros lapos (Érd 20/5) & 2 & $\mathbf{0}$ \\
\hline Augusztus elején érő vadkörte & 1 & $\mathbf{0}$ \\
\hline Árki vadkörte & 3 & 2 \\
\hline Augusztusi hegyes & 2 & 1 \\
\hline Adonyi körte II. & 2 & $\mathbf{0}$ \\
\hline Alma körte & $3 * *$ & $\mathbf{0}$ \\
\hline Alma alakú körte & 2 & $\mathbf{0}$ \\
\hline Alcsevica körte 1 & 2 & $\mathbf{0}$ \\
\hline Alcsevica körte 2 & $\mathbf{0}$ & 1 \\
\hline Augusztusi nagy (Pákozd) & 1 & $\mathbf{0}$ \\
\hline Augusztusi szegfü & $\mathbf{0}$ & $\mathbf{0}$ \\
\hline Augusztusi sárga (31019 Kőszeg) & 1 & $\mathbf{0}$ \\
\hline Ananász & 1 & $\mathbf{0}$ \\
\hline Auróra & 1 & $\mathbf{0}$ \\
\hline Búzás körte (Ráckeve 18/16) & 2 & $\mathbf{0}$ \\
\hline Bálinti őszi körte & 2 & $\mathbf{0}$ \\
\hline B. kisasszony körte & 2 & 1 \\
\hline Bicskei vérbélü & 2 & 2 \\
\hline Bajai bőtermő & 2 & 0 \\
\hline Bókoló kieffer (Leányfalu) & 2 & 3 \\
\hline Búzával érő körte & 2 & $\mathbf{0}$ \\
\hline Bakó János árpával érő (Budaörs) & 2 & 1 \\
\hline Beurse d' & 2 & 1 \\
\hline Brassey Mária (Budaörs 24/4) & $3 * *$ & $\mathbf{0}$ \\
\hline Bosc kobakja & 2 & $\mathbf{0}$ \\
\hline Bajai 6 & $3 * *$ & $\mathbf{0}$ \\
\hline Bőtermő nyári Kálmán & $\mathbf{0}$ & $\mathbf{0}$ \\
\hline Bikedi őszi piros & $\mathbf{0}$ & $\mathbf{0}$ \\
\hline
\end{tabular}

\begin{tabular}{|c|c|c|}
\hline \multirow[b]{2}{*}{ Cultivar } & \multicolumn{2}{|c|}{ Rate of infection* } \\
\hline & $\begin{array}{l}\text { on the } \\
\text { leaves }\end{array}$ & $\begin{array}{l}\text { on the } \\
\text { fruits }\end{array}$ \\
\hline Bikedi nyári körte & 1 & $\mathbf{0}$ \\
\hline Bentler vajkörte (Budaörs 24/2) & 1 & $\mathbf{0}$ \\
\hline Berakó körte & 2 & $\mathbf{0}$ \\
\hline Bonefond asszony & $\mathbf{0}$ & $\mathbf{0}$ \\
\hline Bohusné vajkörtéje & 3 & $\mathbf{0}$ \\
\hline Cinderi körte & 2 & 1 \\
\hline Cornelis (B.őrs) & 2 & $\mathbf{0}$ \\
\hline Cukor körte & 1 & $\mathbf{0}$ \\
\hline Cukor körte (Bicske/Komlós 19/4) & 1 & $\mathbf{0}$ \\
\hline Curé/6 & 1 & $\mathbf{0}$ \\
\hline Cigány körte (Ráckeresztúr) & $\mathbf{0}$ & $\mathbf{0}$ \\
\hline Clapp IX 7/6 & 2 & 1 \\
\hline Cserszegi körte & 2 & 1 \\
\hline Cserlevelücsászár (Budaörs 24/7) & $3 * *$ & $\mathbf{0}$ \\
\hline Csokros muskotály & 2 & $\mathbf{0}$ \\
\hline Császár körte (18/20) & 1 & $\mathbf{0}$ \\
\hline Csákvári nyári körte (30) & 2 & $\mathbf{0}$ \\
\hline Csákvári nyári körte (020) & $\mathbf{0}$ & $\mathbf{0}$ \\
\hline Császár körte Bicske (Komlós) & 1 & $\mathbf{0}$ \\
\hline Cswászár körte & $\mathbf{0}$ & $\mathbf{0}$ \\
\hline Dianna diadala & 2 & $\mathbf{0}$ \\
\hline Dwolinai nyári körte (I. 21/3) & 2 & 1 \\
\hline Decaishe menrich & 3 & $\mathbf{0}$ \\
\hline Dorogmai körte (Noszvaj 20/9) & 1 & $\mathbf{0}$ \\
\hline Duboison vajkörte & 2 & $\mathbf{0}$ \\
\hline Dielszer (Dunabogdány) & 3 & $\mathbf{0}$ \\
\hline Diel vajkörte (Budaörs) & $\mathbf{0}$ & $\mathbf{0}$ \\
\hline Debreceni nagy zöld körte & 2 & $\mathbf{0}$ \\
\hline Erdei vajkörte (Budaörs) & 2 & $\mathbf{0}$ \\
\hline Esperes úri körte & $\mathbf{0}$ & $\mathbf{0}$ \\
\hline Eliz asszony (Budaörs 22/24) & 2 & $\mathbf{0}$ \\
\hline Engheimi vajkörte & 1 & $\mathbf{0}$ \\
\hline Fetel apát kobakja & 2 & 1 \\
\hline Fillér körtéje & 2 & $\mathbf{0}$ \\
\hline Fehérvári körte & 1 & $\mathbf{0}$ \\
\hline Fontenay körte (Budaörs 24/19) & $\mathbf{0}$ & $\mathbf{0}$ \\
\hline Fertőd & $\mathbf{0}$ & $\mathbf{0}$ \\
\hline Gornelis (Budaörs) & 3 & $\mathbf{0}$ \\
\hline Gerjéni nyári körte II. (Gerjén) & 1 & $\mathbf{0}$ \\
\hline Gilles vajkörte (Budaörs) & 2 & 1 \\
\hline Gerjéni vérbél (Gerjén 19/15) & $\mathbf{0}$ & $\mathbf{0}$ \\
\hline George Bousher & 1 & 1 \\
\hline Gatya körte (22/18) & 1 & 1 \\
\hline Gausel bergamottja $(6 / 28)$ & 2 & $\mathbf{0}$ \\
\hline Gortkai körte (Pákozd) & 1 & $\mathbf{0}$ \\
\hline Grand Champion & $\mathbf{0}$ & $\mathbf{0}$ \\
\hline Hosszúréti Ó körte & 1 & $\mathbf{0}$ \\
\hline Hős körte Martonvásár & 3 & $\mathbf{0}$ \\
\hline Hardenpont változat (Dunabogdány) & 2 & $\mathbf{0}$ \\
\hline Hardenpont kései klón & 2 & $\mathbf{0}$ \\
\hline Helyi fajta Hoffmanné (Kismaros) & 1 & $\mathbf{0}$ \\
\hline Héber körte (23/4) & 1 & $\mathbf{0}$ \\
\hline Horváth körte & 1 & $\mathbf{0}$ \\
\hline Hindenburg & 1 & $\mathbf{0}$ \\
\hline Hóka körte & 1 & $\mathbf{0}$ \\
\hline
\end{tabular}




\begin{tabular}{|c|c|c|}
\hline \multirow[b]{2}{*}{ Cultivar } & \multicolumn{2}{|c|}{ Rate of infection* } \\
\hline & $\begin{array}{l}\text { on the } \\
\text { leaves }\end{array}$ & $\begin{array}{l}\text { on the } \\
\text { fruits }\end{array}$ \\
\hline Július végén érő (Délegyháza) & 2 & 1 \\
\hline Jún. végén, júl. elején érő (19/17) & 1 & 1 \\
\hline Júliusi esperes & 3 & $\mathbf{0}$ \\
\hline Juhászné körtéje & 3 & $\mathbf{0}$ \\
\hline Júl. végén, aug. elején érő $8 / 20$ & 3 & 1 \\
\hline Jó Sarolta & 2 & $\mathbf{0}$ \\
\hline Jó Szürke & 2 & $\mathbf{0}$ \\
\hline Júliusi arany (M.V.) & $\mathbf{0}$ & $\mathbf{0}$ \\
\hline Jégkörte & $\mathbf{0}$ & $\mathbf{0}$ \\
\hline Jászteleki nyári körte & 1 & $\mathbf{0}$ \\
\hline Kerek körte & 3 & 1 \\
\hline Kurva körte (Pákozd) & 3 & 3 \\
\hline Kápolnai körte & 2 & 3 \\
\hline Kisszegf körte (Budaörs 24/13) & 2 & $\mathbf{0}$ \\
\hline Körte 1/5 (Ráckeve 22/11) & 2 & $\mathbf{0}$ \\
\hline Klára trió (Budaörs 24/6) & 1 & $\mathbf{0}$ \\
\hline Körte 1/4 (Ráckeve 22/12) & 1 & $\mathbf{0}$ \\
\hline Kongresszus körte (Becsehely) & 2 & 1 \\
\hline Kongresszus körte & 1 & $\mathbf{0}$ \\
\hline Korai szagos körte & $\mathbf{0}$ & $\mathbf{0}$ \\
\hline Körte 2/5 (Ráckeve 22/6) & 2 & $\mathbf{0}$ \\
\hline Körte 2/9 (Ráckeve 22/6) & 1 & $\mathbf{0}$ \\
\hline Károlyi körte (31014 Kőszeg) & 3 & $\mathbf{0}$ \\
\hline Körte B/1 & 3 & 1 \\
\hline Kieffer körte (Érd-Ófalu 19/22) & 1 & $\mathbf{0}$ \\
\hline Körte 1/3 (Ráckeve 22/9) & 1 & $\mathbf{0}$ \\
\hline Kései bosc 14/2 (Sárospatak 23/1) & 2 & $\mathbf{0}$ \\
\hline Korai vérbél (Bicske 19/9) & 0 & $\mathbf{0}$ \\
\hline Körte 2/7 (Ráckeve 22/5) & 1 & $\mathbf{0}$ \\
\hline Késői bőr (Pákozd) & $\mathbf{0}$ & $\mathbf{0}$ \\
\hline Kelenvölgyi pirosbél & $\mathbf{0}$ & $\mathbf{0}$ \\
\hline Kakas körte & 2 & $\mathbf{0}$ \\
\hline Körte B/2 (18/2) & $\mathbf{0}$ & $\mathbf{0}$ \\
\hline Körte 2/10 Ráckeve (22/16) & 3 & $\mathbf{0}$ \\
\hline Köcsög körte Vk3 & 2 & 3 \\
\hline K 28 & 2 & 3 \\
\hline K 35 & 1 & $\mathbf{0}$ \\
\hline K 36 & 1 & $\mathbf{0}$ \\
\hline K 63 & $\mathbf{0}$ & $\mathbf{0}$ \\
\hline K 66 & $\mathbf{0}$ & $\mathbf{0}$ \\
\hline K 67 & 1 & $\mathbf{0}$ \\
\hline K 69 & $\mathbf{0}$ & $\mathbf{0}$ \\
\hline Kieffer II (20/18) & $\mathbf{0}$ & $\mathbf{0}$ \\
\hline Kurva körte (fojtós P. Kismarót) & 2 & $\mathbf{0}$ \\
\hline Körte 1/7 (Ráckeve P) & 3 & $\mathbf{0}$ \\
\hline $\mathrm{K} 14$ & 2 & 3 \\
\hline Lapos vajk.(aug.elején, közepén érö 18/23) & 3 & $\mathbf{0}$ \\
\hline Leányfalusi piros & 1 & $\mathbf{0}$ \\
\hline Lincoln körte (18/1) & 2 & $\mathbf{0}$ \\
\hline Lőrinc körte (29/9) & 3 & $\mathbf{0}$ \\
\hline Liegel téli vajkörte & $\mathbf{0}$ & $\mathbf{0}$ \\
\hline Ledesz Amália (Budaörs P Ledesz) & 3 & $\mathbf{0}$ \\
\hline Lejtő körte (11/2) & 3 & 1 \\
\hline Monchallard Dunabogdány & 1 & $\mathbf{0}$ \\
\hline Mézes körte (Budaörs 22/25) & 1 & $\mathbf{0}$ \\
\hline
\end{tabular}

\begin{tabular}{|c|c|c|}
\hline \multirow[b]{2}{*}{ Cultivar } & \multicolumn{2}{|c|}{ Rate of infection* } \\
\hline & $\begin{array}{l}\text { on the } \\
\text { leaves }\end{array}$ & $\begin{array}{l}\text { on the } \\
\text { fruits }\end{array}$ \\
\hline Mosoly körte & 2 & $\mathbf{0}$ \\
\hline Méznár körte & 1 & $\mathbf{0}$ \\
\hline Mandula vajkörte & 2 & $\mathbf{0}$ \\
\hline Móri császárkörte & 2 & $\mathbf{0}$ \\
\hline Márianosztrai körte 1 & 1 & 0 \\
\hline Montrenili & 2 & $\mathbf{0}$ \\
\hline Montreál vajkörte (Budaörs) & 2 & $\mathbf{0}$ \\
\hline Márianosztrai körte 2 & 2 & $\mathbf{0}$ \\
\hline Moon Glou & $3 * *$ & 1 \\
\hline Marianna & 3 & 1 \\
\hline Miklós körte & 1 & $\mathbf{0}$ \\
\hline Mézes körte (Szentlörinc) & $\mathbf{0}$ & $\mathbf{0}$ \\
\hline Nácika körte (23/5) & 2 & $\mathbf{0}$ \\
\hline Nagyasszony körte & $\mathbf{0}$ & $\mathbf{0}$ \\
\hline N 310 & 2 & 1 \\
\hline Napoca & 2 & 0 \\
\hline Nácika II. & 2 & $\mathbf{0}$ \\
\hline Nyári körte (99 Kieffer vajkörte) & 3 & 1 \\
\hline Nyári körte (Nagytétény 22/1) & 2 & $\mathbf{0}$ \\
\hline Nyári körte (Gerjén 19/13) & 1 & $\mathbf{0}$ \\
\hline Nyári piros körte (Gerjén 19/14) & 2 & $\mathbf{0}$ \\
\hline Nyári Kálmán (Érd 20/4) & $\mathbf{0}$ & $\mathbf{0}$ \\
\hline Nyári nyakas körte (Bicske) & 2 & $\mathbf{0}$ \\
\hline Nyári clapp (Budaörs 23/23) & 3 & 1 \\
\hline Nyári bergamott & 3 & $\mathbf{0}$ \\
\hline Nyárig tartó (6/19) & 2 & $\mathbf{0}$ \\
\hline Nyári zöld kobak & $\mathbf{0}$ & $\mathbf{0}$ \\
\hline Nyári körte (Mátyás) & 2 & $\mathbf{0}$ \\
\hline Nyári esperes & 3 & 1 \\
\hline Orient & 2 & 1 \\
\hline Olasz császár (Budaörs 24/3) & 1 & $\mathbf{0}$ \\
\hline Olasz 4 & 2 & $\mathbf{0}$ \\
\hline Őszi körte II. (Kieffer vajkörte) & 2 & $\mathbf{0}$ \\
\hline Őszi rozsdás körte & 2 & 1 \\
\hline Őszi rozsdás Szentlőrinc & $\mathbf{0}$ & $\mathbf{0}$ \\
\hline Öszi körte (100 Szekszárd 24/25) & 3 & 1 \\
\hline Öszi parázs körte & 1 & 2 \\
\hline Őszi pálinka 2 & 3 & $\mathbf{0}$ \\
\hline Ötvös körte & 3 & $\mathbf{0}$ \\
\hline Öszi vajkörte & 1 & $\mathbf{0}$ \\
\hline Öszi vajkörte (I.b.) & $3 * *$ & $\mathbf{0}$ \\
\hline Őszi cukor & 3 & $\mathbf{0}$ \\
\hline Papkörte (Bicske/Komlós/19/1) & 2 & $\mathbf{0}$ \\
\hline Piros nyári körte (Bicske) & 1 & $\mathbf{0}$ \\
\hline Pöttyös körte & $\mathbf{0}$ & $\mathbf{0}$ \\
\hline Pomázi füge körte & 1 & $\mathbf{0}$ \\
\hline $\mathrm{Pb} 242$ & $\mathbf{0}$ & $\mathbf{0}$ \\
\hline Piros búzás körte & 3 & 1 \\
\hline Papkörte bőtermő (23/1) & 2 & $\mathbf{0}$ \\
\hline $\mathrm{Pb} 299$ & $\mathbf{0}$ & $\mathbf{0}$ \\
\hline Párizsi gróf (Budaörs 23/21) & 2 & $\mathbf{0}$ \\
\hline Piros bél óriás (Bicske/Komlós) & 1 & $\mathbf{0}$ \\
\hline P 2 Pettend & 1 & $\mathbf{0}$ \\
\hline P 3 Pettend & 3 & $\mathbf{0}$ \\
\hline Pomázi nyári körte II. & 3 & 1 \\
\hline
\end{tabular}




\begin{tabular}{|c|c|c|}
\hline \multirow[b]{2}{*}{ Cultivar } & \multicolumn{2}{|c|}{ Rate of infection* } \\
\hline & $\begin{array}{l}\text { on the } \\
\text { leaves }\end{array}$ & $\begin{array}{l}\text { on the } \\
\text { fruits }\end{array}$ \\
\hline Péter bátya $(8 / 90 \mathrm{P})$ & 3 & $\mathbf{0}$ \\
\hline Pápai körte & $\mathbf{0}$ & $\mathbf{0}$ \\
\hline Papkörte igen bőtermő (Sárospatak) & 3 & $\mathbf{0}$ \\
\hline Pessert Maya & 1 & $\mathbf{0}$ \\
\hline Rózsavölgyi 6 & 3 & $\mathbf{0}$ \\
\hline Solani körte & 2 & $\mathbf{0}$ \\
\hline Spadónia téli körte (Törökbálint) & 3 & $\mathbf{0}$ \\
\hline Salangue Panache (Budaörs 23/24) & 2 & $\mathbf{0}$ \\
\hline Sárkörte & 3 & $\mathbf{0}$ \\
\hline Sándor körte (Noszvaj 20/18) & 2 & $\mathbf{0}$ \\
\hline Steiner körte & $\mathbf{0}$ & $\mathbf{0}$ \\
\hline Stuttgarti pirok & $\mathbf{0}$ & $\mathbf{0}$ \\
\hline Solymári cukor körte & 2 & $\mathbf{0}$ \\
\hline Szagos körte (aug. közepén érő) & 2 & $\mathbf{0}$ \\
\hline Szagos körte 105 Kieffer vajkörte & 2 & 1 \\
\hline Szentlőrinc 6 & 2 & 1 \\
\hline Szentendrei császár & 2 & 1 \\
\hline Szeptemberi óriás & 2 & $\mathbf{0}$ \\
\hline Szücsi őszi körte & 2 & $\mathbf{0}$ \\
\hline SZU-1 & 1 & 2 \\
\hline Szentesi körte & 3 & $\mathbf{0}$ \\
\hline Szeptemberi piros (Érd 20/3) & $\mathbf{0}$ & $\mathbf{0}$ \\
\hline Szücsi vadkörte I. & $\mathbf{0}$ & $\mathbf{0}$ \\
\hline Szekszárdi bergamott & $\mathbf{0}$ & $\mathbf{0}$ \\
\hline Szőke körte & 1 & $\mathbf{0}$ \\
\hline Szombatfai sózó körte & $\mathbf{0}$ & $\mathbf{0}$ \\
\hline Szászbereki vajkörte (382) & 2 & $\mathbf{0}$ \\
\hline Tallér körte & 2 & 1 \\
\hline Téli zöld körte (19/20) & $\mathbf{0}$ & 0 \\
\hline Téli Kálmán & 1 & $\mathbf{0}$ \\
\hline Talpas körte & 1 & $\mathbf{0}$ \\
\hline Tehénláb körte Nagydorog & $\mathbf{0}$ & $\mathbf{0}$ \\
\hline Téli körte & $\mathbf{0}$ & $\mathbf{0}$ \\
\hline Tongre (Budaörs) & 1 & $\mathbf{0}$ \\
\hline Vilmos körte (Bicske/Komlós) & 2 & $\mathbf{0}$ \\
\hline Viki körte & 1 & $\mathbf{0}$ \\
\hline Volkmarsoni vajkörte & 3 & 1 \\
\hline Vadkörte (nyári körte 179) & 2 & $\mathbf{0}$ \\
\hline Virgonkuse (Budaörs 22/23) & $\mathbf{0}$ & $\mathbf{0}$ \\
\hline Váraljai császár óriás & 2 & $\mathbf{0}$ \\
\hline Váraljai nyári 1 & 3 & $\mathbf{0}$ \\
\hline Vilmos & 1 & 0 \\
\hline Vilmos császár körte Noszvaj & 1 & $\mathbf{0}$ \\
\hline Vérteskozmai körte (060) & 2 & $\mathbf{0}$ \\
\hline Váli körte 2 & 1 & $\mathbf{0}$ \\
\hline Váli körte & 3 & $\mathbf{0}$ \\
\hline Verténé körtéje (6/9) & 1 & $\mathbf{0}$ \\
\hline Vérteskozmai körte (040) & 2 & $\mathbf{0}$ \\
\hline Vanquelin & 1 & 0 \\
\hline Wienne diadala (Budaörs 20/1) & 2 & $\mathbf{0}$ \\
\hline Zabbal érö (4/9) & 1 & $\mathbf{0}$ \\
\hline Zánkai körte magonc & 3 & $\mathbf{0}$ \\
\hline Zölden érő kúpos (Pákozd) & $\mathbf{0}$ & $\mathbf{0}$ \\
\hline Zsámbéki körte 3 & 1 & $\mathbf{0}$ \\
\hline 3-38-TA & 1 & $\mathbf{0}$ \\
\hline
\end{tabular}

\begin{tabular}{|c|c|c|}
\hline \multirow[b]{2}{*}{ Cultivar } & \multicolumn{2}{|c|}{ Rate of infection* } \\
\hline & $\begin{array}{l}\text { on the } \\
\text { leaves }\end{array}$ & $\begin{array}{l}\text { on the } \\
\text { fruits }\end{array}$ \\
\hline 3-51-TA & 3 & 1 \\
\hline 3-54-TA & 3 & 1 \\
\hline 3-30-TA & $3^{* * *}$ & 1 \\
\hline 30017 Fertőd (21/12) & 3 & 1 \\
\hline 30061 Fertőd (21/10) & 2 & $\mathbf{0}$ \\
\hline 30029 Fertöd (21/14) & 1 & $\mathbf{0}$ \\
\hline 30024 Mézkörte & 1 & $\mathbf{0}$ \\
\hline 0632 & $\mathbf{0}$ & $\mathbf{0}$ \\
\hline 0629 & 2 & $\mathbf{0}$ \\
\hline 3-32-TA & 2 & $\mathbf{0}$ \\
\hline 30060 Fertőd (21/7) & 3 & $\mathbf{0}$ \\
\hline II. A4 (15/2) & $\mathbf{0}$ & $\mathbf{0}$ \\
\hline \begin{tabular}{|l|}
$96-165$ \\
\end{tabular} & $\mathbf{0}$ & $\mathbf{0}$ \\
\hline II. B3 (6/4) & $\mathbf{0}$ & $\mathbf{0}$ \\
\hline \begin{tabular}{|l|} 
II. A4 $1 / 2$ \\
\end{tabular} & 1 & $\mathbf{0}$ \\
\hline $9 / 8$ & 1 & $\mathbf{0}$ \\
\hline $4 / 6$ & 1 & $\mathbf{0}$ \\
\hline
\end{tabular}

*Rate of infection (scale): 0 = free of infection, $1=$ slight infection, $2=$ medium infection, $3=$ strong infection

**Extremely high infestation was detected (infection was much higher than 70 , sometimes as high as 100 per cent).

Table 2. Relative sensitivity of leaves and fruits of European pear genotypes (commercial cultivars, conventional land varieties, registered local clones, seedlings, wild seedlings) to field infection by Venturia pirina ('Újfehértó' pear gene bank, Hungary)

\begin{tabular}{|c|c|c|c|c|}
\hline \multirow{2}{*}{$\begin{array}{c}\text { Trend of infection } \\
\text { on leaves as } \\
\text { compared to fruits }\end{array}$} & \multicolumn{2}{|c|}{$\begin{array}{l}\text { Rate of infection } \\
\text { according to the } \\
\text { relative susceptibility } \\
\text { (categories } 0,1,2 \text { and } 3)^{*}\end{array}$} & \multirow{2}{*}{$\begin{array}{l}\text { Per cent } \\
\text { ratio of } \\
\text { cultivars } \\
\text { involved }\end{array}$} & \multirow{2}{*}{$\begin{array}{c}\text { Total } \\
\text { per } \\
\text { cent of } \\
\text { cultivars } \\
\text { involved }\end{array}$} \\
\hline & $\begin{array}{l}\text { on the } \\
\text { leaves }\end{array}$ & $\begin{array}{l}\text { on the } \\
\text { fruits }\end{array}$ & & \\
\hline Free of infection & 0 & 0 & $18.0 \%$ & $18.0 \%$ \\
\hline \multirow{3}{*}{$\begin{array}{l}\text { Similar rate of } \\
\text { infection on leaves } \\
\text { and fruits }\end{array}$} & 1 & 1 & $1.1 \%$ & \multirow{3}{*}{$2.2 \%$} \\
\hline & 2 & 2 & $0.7 \%$ & \\
\hline & 3 & 3 & $0,4 \%$ & \\
\hline \multirow{6}{*}{$\begin{array}{l}\text { Higher rate of } \\
\text { infection on leaves } \\
\text { than on fruits }\end{array}$} & 1 & 0 & $23.5 \%$ & \multirow{6}{*}{$76.9 \%$} \\
\hline & 2 & 0 & $25.3 \%$ & \\
\hline & 3 & 0 & $14.0 \%$ & \\
\hline & 2 & 1 & $7.2 \%$ & \\
\hline & 3 & 1 & $6.5 \%$ & \\
\hline & 3 & 2 & $0.4 \%$ & \\
\hline \multirow{3}{*}{$\begin{array}{l}\text { Higher rate of } \\
\text { infection on fruits } \\
\text { than on leaves }\end{array}$} & 0 & 1 & $0.4 \%$ & \multirow{3}{*}{$2.9 \%$} \\
\hline & 1 & 2 & $0.7 \%$ & \\
\hline & 2 & 3 & $1.8 \%$ & \\
\hline
\end{tabular}

*Rate of infection: 0 . Tolerant (free of infection), 1. Slightly susceptible (slight infection is typical), 2. Susceptible (slight or medium infection is typical), 3. Highly susceptible (strong infection is typical)

Results clearly show that substantial differences have been detected in the rate of pear scab infection of leaves and fruits (Table 1). Conspicuously some 18 per cent of the inspected genotypes remained completely free of infection in the years with weather favouring to this disease because both the leaves and the fruits remained healthy. On the other hand, 
Table 3. Distribution of European pear genotypes (commercial cultivars, conventional land varieties, registered local clones, seedlings, wild seedlings) according to their field sensitivity to Venturia pirina infection ('Újfehértó' pear gene bank, Hungary)

\begin{tabular}{|l|c|c|}
\hline \multirow{2}{*}{ Rate of infection (scale) } & \multicolumn{2}{|c|}{$\begin{array}{c}\text { Per cent distribution of } \\
\text { inspected European pear } \\
\text { genotypes }\end{array}$} \\
\cline { 2 - 3 } & $\begin{array}{r}\text { on the leves } \\
\text { on the fruits }\end{array}$ \\
\hline $\begin{array}{l}\text { a) Generally free of infection } \\
\text { (tolerant) }\end{array}$ & $18.6 \%$ & $81.1 \%$ \\
\hline $\begin{array}{l}\text { b) Generally slightly infected } \\
\text { (slightly susceptible) }\end{array}$ & $27.5 \%$ & $15.2 \%$ \\
\hline $\begin{array}{l}\text { c) Generally medium infection is typical } \\
\text { (susceptible) }\end{array}$ & $34.2 \%$ & $1.5 \%$ \\
\hline $\begin{array}{l}\text { d) Generally strong infection is typical } \\
\text { (highly susceptible) }\end{array}$ & $19.7 \% *$ & $2.2 \%$ \\
\hline
\end{tabular}

*Extremely high infection (much higher than 50, sometimes as high as 100 per cent) was typical in the case of 2.6 per cent of the inspecwted genotypes

$78 \%$ of the investigated genotypes were infected in some or more extent (Table 2). The rate of infection was similar (1-1 to 3-3) of leaves and fruits at some $2.2 \%$ of the genotypes only but the infection was different on leaves and fruits at $79.8 \%$ of the genotypes inspected (Table 2). In the case of the majority of these genotypes $(76.9 \%)$ the rate of infection was higher on leaves than on fruits (Table 2). The opposite situation - higher infection on fruits than on leaves - appeared in the case of a small number (2.9\%) of the genotypes only (Table 2); these genotypes were: Bókoló kieffer (Leányfalu), Kápolnai körte, Köcsög körte Vk3, K 28, K 14, Öszi parázs körte, SZU-1. Some genotypes were extremely sensitive to leaf infection because in years with favourable weather to disease incidence it was much higher than 70 , sometimes as high as 100 per cent: Alma körte, Brassey Mária, Bajai 6. Cserlevelü császár, Moon Glou, Öszi vajkörte, 3-30-TA. Notwithstanding, in spite of the slight leaf infection $(23.5 \%$ of inspected genotypes), medium leaf infection (25.3\% of inspected genotypes) or what is more strong infection on leaves (14.0\% of inspected genotypes) as much as $72.8 \%$ of the investigated genotypes have shown no infection on fruits (Table 2).

The distribution of the investigated 271 European pear genotypes according to their sensitivity to pear scab infection is demonstrated in Table 3. Some one fifth $(18.6 \%)$ of the genotypes inspected was tolerant to leaf infection and their majority $(81.1 \%)$ was tolerant to fruit infection. Also some one fifth of the genotypes $(19.7 \%)$ were highly susceptible to leaf infection but only minor rate of them $(2.2 \%)$ were highly susceptible to fruit infection (Table3).The rest of the genotypes were slightly or moderately susceptible on leaves $(61.7 \%)$ or on fruits $(16.7 \%)$. So the sensitivity of genotypes to pear scab infection has shown close to normal distribution. There were 44 genotypes that have remained completely free of infection even in the years with favourable weather to pear scab infections (see in Table4).
Table 4. European pear genotypes being tolerant or slightly susceptible to Veturia pirina field infection, after research made in Hungary

\begin{tabular}{|c|c|c|}
\hline $\begin{array}{c}\text { Scarcely } \\
\text { susceptible } \\
\text { after Husz (1941) }\end{array}$ & $\begin{array}{l}\text { Maximum field } \\
\text { infection is always } \\
\text { less than } 10 \text { per cent } \\
\text { after Szabó and } \\
\text { Soltész (1998) }\end{array}$ & $\begin{array}{c}\text { Both the leaves and } \\
\text { the fruits remained } \\
\text { completely free of } \\
\text { field infection during } \\
\text { nine years' period of } \\
\text { observations } \\
\text { (Benedek, Szabó and } \\
\text { Nyéki: present study) }\end{array}$ \\
\hline $\begin{array}{l}\text { Bosc kobak** } \\
\text { Clairgeau vajkörte } \\
\text { Papkörte** } \\
\text { Serres Olivér }\end{array}$ & $\begin{array}{l}\text { Aratási cukor } \\
\text { Árpával érő } \\
\text { Bikezdi nyári* } \\
\text { Bőr körte } \\
\text { Citron des Carmes } \\
\text { Füzér körte } \\
\text { Kieffer javított } \\
\text { Mezőkövesdi } 5 \\
\text { Nyári piros körte** } \\
\text { Piros búzás körte*** } \\
\text { Pomázi nyári körte*** } \\
\text { Republica } \\
\text { Szentendrei császár** } \\
\text { Váraljai nyári*** } \\
\text { Vérbél } \\
\text { Vérbél bőtermő } \\
\text { Zabbal érő* }\end{array}$ & 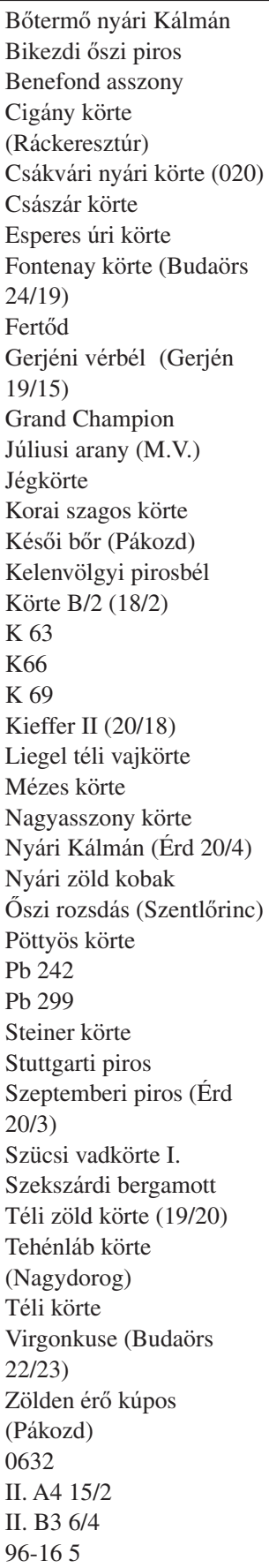 \\
\hline
\end{tabular}

* Only slight infection appeared in the study of Benedek, Szabó and Nyéki (present study)

** Medium infection appeared in the study of Benedek, Szabó and Nyéki (present study)

*** Strong infection appeared in the study of Benedek, Szabó and Nyéki (present study) 


\section{Discussion and conclusions}

European pear cultivars showing no or minor rate of symptoms under field conditions to pear scab after natural or artificial inoculation are considered to be practically tolerant to this disease in the literature (see for example Szabó and Soltész, 1989; Postman et al., 2004; Villata et al., 2005). In this study as much as 44 genotypes $(18 \%)$ were found to be tolerant of the 271 ones inspected (Table 4).

Taking earlier information into account the number of genotypes being more or less tolerant to pear scab under the climatic conditions in Hungary is as much as 66 (Table 4) because Husz (1941) mentioned 5 and Szabó and Soltész (1989) have found 17 additional cultivars being scarcely susceptible to this pathogen besides the 44 tolerant genotypes having been discovered in the present study. Some of these genotypes may be suitable to commercial pear growing with highly reduced chemical control against pear scab; however, besides their tolerance to pear scab their yield capacity and fruit quality should be evaluated.

Genotypes having shown good field tolerance to pear scab infection in field studies in Hungary (Table 4) should be further studied under field conditions and also in glasshouse trials in the future to check if they show any hypersensitive reactions after leaf infections that Chevalier et al. (2011) detected in he case of the cultivar 'Navara' known to be resistant to this pathogen. Also the presence of the resistance gene discovered by Bouvier et al. (2012) in the resistant cultivar 'Navara' could be checked up in these genotypes because they could serve as useful material in breeding pear cultivars resistant to pear scab.

In this study great differences have been shown between the sensitivity of leaves and fruits to pear scab infections in the case of most European pear genotypes studied (Tables $1-3)$. So less effort is needed in commercial pear growing to keep fruits free of scab infection than the leaves. This finding may be useful in commercial pear production.

\section{Acknowledgement}

Research was sponsored by TECH_08-A3/2-2008-0373 and TECH_08-A4/2-2008-0138 grants.

\section{References}

Brewer, L.R., Alspach, P.A., Morgan, C. \& Bus, V.G.M. (2009): Resistance to scab caused by Venturia pirina in interspecific pear (Pyrus spp.) hybrids. New Zealand J. Crop and Hort.Sci., 37. (3): 211-218.

Bouvier, L., Bourcy, M., Tellier, M., Guérif, P., Denancé, C., Durel, C-E. \& Lespinasse, Y. (2012): A new scab resistance gene Rvp1 from the European pear cultivar 'Navara' map in a genomic region synthetic to an apple scab resistance gene cluster on linkage group 2. Tree Genetics \& Genomes, 8: 53-60.

Chevalier, M., Tellier, M., Lespinasse, Y. \& Mallet, R. (2011): Microscopic studies of susceptibility and resistance symptoms expressed on two cultivars of Pyrus communis inoculated with Venturia pirina . Acta Horticilturae (ISHS), 909: 471-477.

Husz B. (1941): A beteg növény és gyógyítása. Term. Tud. Társulat, Budapest: pp. 343.

Postman J., Spotts, R. \& Calabro, J. (2004): Pear scab resistance in Pyrus germplasm. Pear International Symposium, February 1-5, 2004, Stellenbossch, South Africa: 38.

Szabó T. \& Soltész M. (1998): Susceptibility to diseases and pests of pear varieties in Hungary. Acta Horticulturae (ISHS), 475: 497502.

Villalta, O.N., Washington, W.S., McGregor, G.R., Richards, S.M. \& Liu, S.M. (2005): resistance to pear scab in European and Asian pear cultivars in Australia. Acta Horticulturae (ISHS), 694: 129-132. 